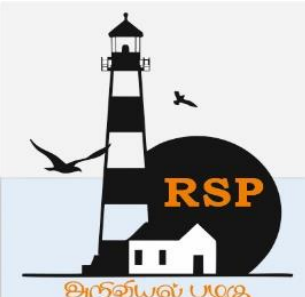

INTERNATIONAL RESEARCH JOURNAL ON ADVANCED SCIENCE HUB

-ISSN : $2582-4376$ Open Access

RSP SCIENCE HUB

(The Hub of Research Ideas)

Available online at www.rspsciencehub.com

\title{
Optimization in Micro aerial Vehicle for Higher Performance
}

A.J Sriganapathy ${ }^{1}$, Gunasekaran $R^{2}, T$. Kalaiarasan $N^{3}$, Balabharathi $R^{4}$, Haridas $T^{5}$

${ }^{1}$ Assistant professor, Department of Aeronautical Engineering, Mahendra institute of engineering and technology, Tiruchengode, Namakkal. Tamilnadu, India.

2.3.4.5 UG Scholar, Department of Aeronautical Engineering, Mahendra institute of engineering and technology, Tiruchengode, Namakkal. Tamilnadu, India.

Sri.aero87@gmail.com ${ }^{1}$,aeroguna2017@gmail.com ${ }^{2}$, kalaiarasan21099@gmail.com ${ }^{3}$,

balabharathi436@gmail.com ${ }^{4}$,hariaero147@gmail.com ${ }^{5}$

\begin{abstract}
Micro Air Vehicle (MAV) is the current research and development work worldwide. This work describes the design and optimization method of Micro Air Vehicles. Here the optimization method to minimize the vehicle size, the objective function for this optimization problem, for the set of design variables is found from the aerodynamic analysis. This aerodynamic analysis is mainly based on the results obtained from the wind tunnel. Optimization for improving the endurance is explained in the similar way as the optimization problem of minimizing the size of the vehicle with the slight change in the objective function.
\end{abstract}

Key words: Optimization of MAV, Minimize size, Maximum endurance.

\section{Introduction}

MAVs that fly using flapping wings hold great potential for indoor reconnaissance and hovering observation. The objective of this work is to build a small flapping wing vehicle that possesses a wingspan of approximately $1 \mathrm{ft} .(30 \mathrm{~cm})$ and weighs approximately one ounce $(28 \mathrm{~g})$. This vehicle should be capable of vertical takeoff and landing (VTOL) as well as high manoeuvrability for indoor environments. The aerodynamics of the Quad-Wing Vehicle, a Micro Air Vehicle designed to hover with four flapping wings in a dragonflylike configuration, is investigated using Computational Fluid Dynamics (CFD), potential flows analysis, and experimental testing. The CFD analysis investigates the kinematics-parameters design space and identifies values of kinematics parameters that maximize the vertical force production in hovering mode while minimizing the aerodynamic power requirement. It also investigates other important considerations, such as the effect of scaling, multi-wing interactions, and comparison with other flapping configurations. In the potential flows analysis, an unsteady 2D panel code is developed and compared with CFD for a broad range of hoveringflight simulations.[1-4] The results show that, with further development, panel codes may be useful to designers of hovering flapping MAVs because of their time-saving potential compared to CFD. The experimental testing focuses on isolating the aerodynamic forces from other measured forces on a bench top flapping device, and the findings of the experimental study will be useful for later researchers using experimental methods to study flapping MAV aerodynamics. we discuss an approach we used to design flapping wing Micro Air Vehicles (MAV). The approach makes use of the conventional precision machining methods, such as Rapid Prototyping 3D printing, Electrical Discharge Machining and Laser Micromachining techniques, to manufacture the 


\section{www.rspsciencehub.com}

MAV parts. The current generation of MAV's has a total weight of 10.8 grams (12.56 grams with one Camera) and a span of $20 \mathrm{~cm}$ and it can achieve a payload of around 3 grams. The MAV's not only are capable of performing simple acrobatic maneuver in the air but also can hover in place with very little drift. We are in the process of redesigning the next generation of MAV's, which not only offer a reduction in size but also make use of vision-based tracking technique to perform obstacle-avoidance flight adjustments. There are at least eight inter-connected scientific disciplines within the MAV design trade space \& structures, aerodynamics, navigation, feedback \& control, materials, sensors \& actuators, propulsion \& power, and communications. Because the size, shape, weight and aerodynamic restrictions imposed on the vehicles, all these areas are intertwined among one another and therefore a system consideration of the total design cannot be shortcut even at the earlier stage of the design cycle. The research for an autonomous MAV is just begun, what was described here is only the tip of the iceberg. There are still many unexplored areas needing attention if our final goal is to mimic the nature such that an MAV can be as agile as a dragon fly or a fruit fly. [4-8].

\section{Modelling of MAV's}

The group sifted through the literature to gain insight into the motion of flapping wings and how they generate lift. The modeling process began with a basic idea of what the group was hoping to achieve with this work - a relatively small flapping wing vehicle capable of vertical takeoff and landing, hover, and horizontal flight. Through research, that it was decided that mimicking a two-wing bird or insect would be rather difficult and unreasonable, for these animals move in a very complex manner. Through more research and the discovery of the clap-and-fling method of flight, the group decided upon a four-wing vehicle with tail surfaces to provide stability and control. This would be easier to model and manufacture than the motion required for a two-wing vehicle to take flight. As the group continued to model the MAV, the next step was to choose which components and materials would be used in the design. In the development of the body, and wings, the group researched materials that were lightweight, yet durable enough to withstand impact and rapid movement. Also involved in the design are several electronic components: a battery and adapter, a motor and speed controller, three actuators, a receiver, and a transmitter. In this work the body weight is a significant design constraint, the group eliminated metals as an option for the body material and instead decided to research composites and polymers. The group eventually narrowed the field to two different materials. The first, balsa wood, had been used in previous flapping wing MAVs. The group also found a polymer, Polymethacrylimide foam, that is often used in sandwich construction and less dense than balsa wood. For a design involving four wings, it is crucial for each wing to be as lightweight as possible. For this purpose, the group decided to research thin polyester films to comprise the wing, and lightweight spars to provide support and allow for the clap and fling effect. A hinge is necessary to hold the four wings together and allow a flapping motion that brings the leading edges together. The group originally chose aluminum 6061 as a material. The motion of the hinge will be created by a drive train connected to the motor. The design of model is shown in fig.1,2 \&3. 

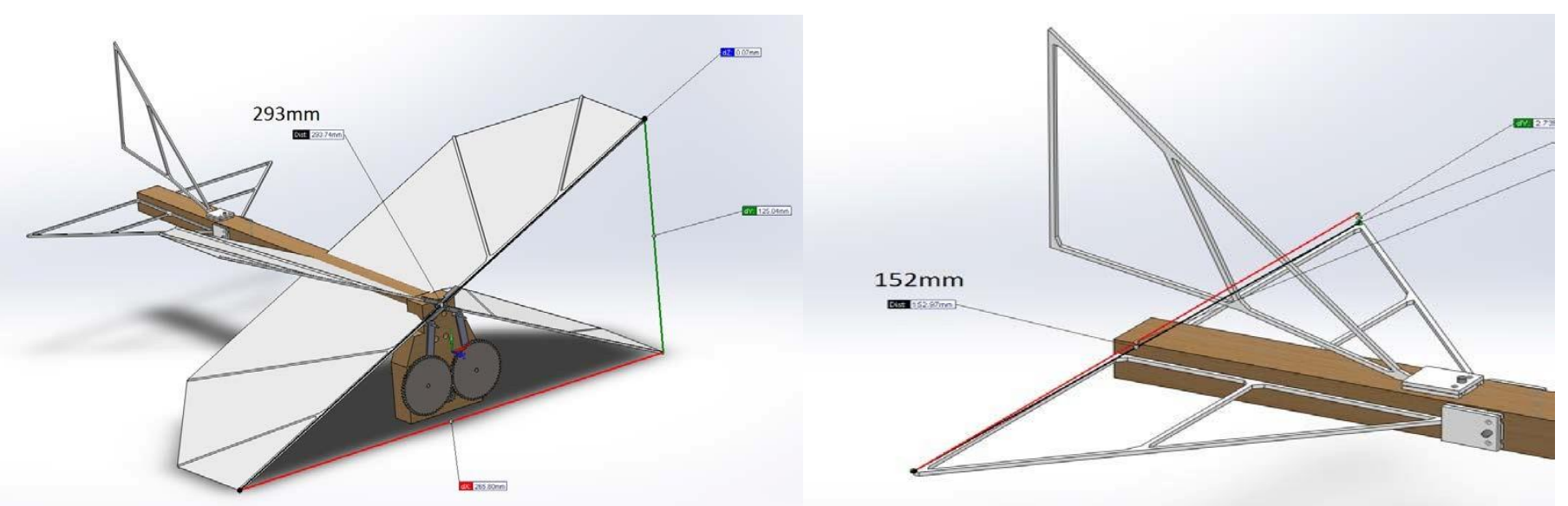

Fig.1.Isometric View of the SolidWorks Model

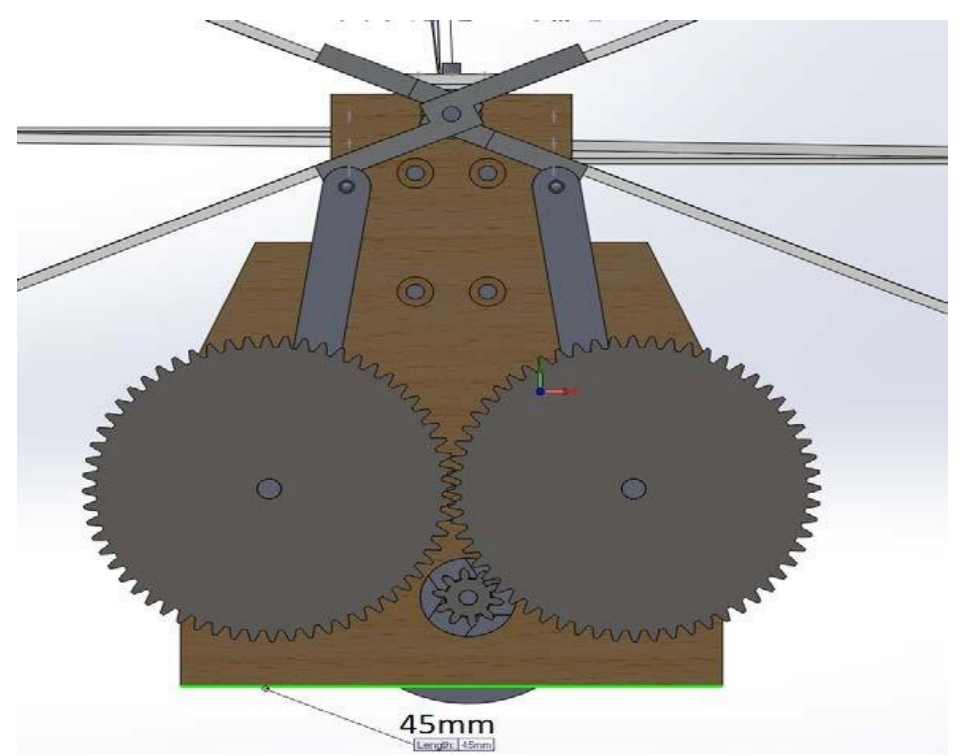

Fig.3-Finalized Drive Train
Fig.2.SolidWorks CAD Model of Tail Structure

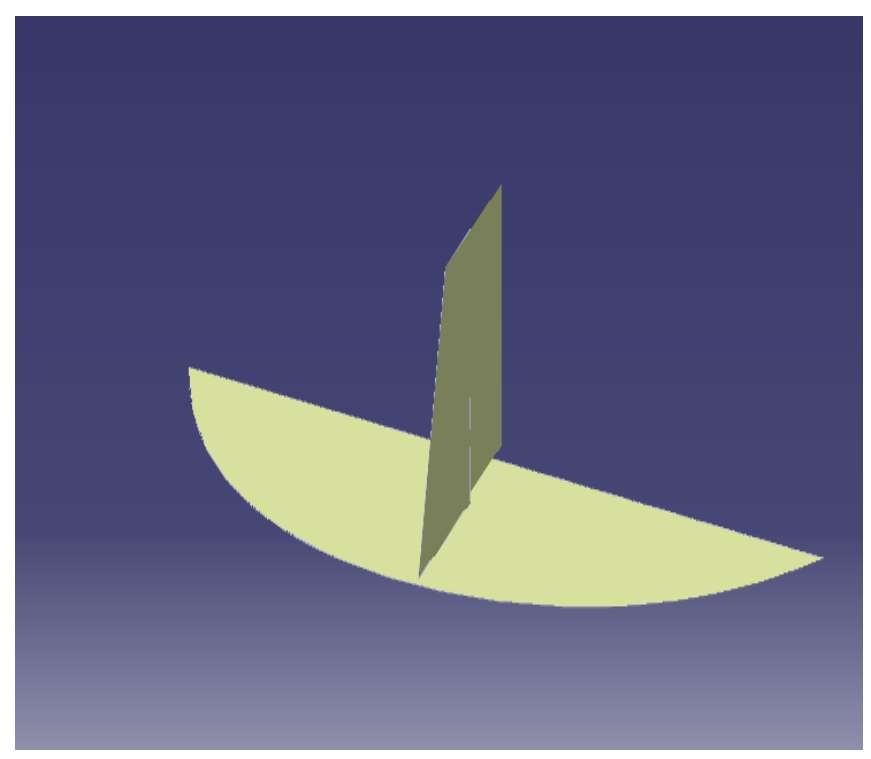

Fig.4.- Catia diagram of delfly

\section{Preprocessing Modelling:}

Fluent is a computational fluid dynamics computer code developed and marketed by Fluent Inc. The code solves the equations for conservation of mass, momentum, energy and other relevant fluid variables using a cell-centred finite-volume method. FLUENT requires high quality mesh to avoid numerical diffusion. Several Mesh Quality Metrics are involved in order to quantify the quality; The aspect ratio and cell size change mesh metrics are also very important. Generally try to keep maximum skewness of volume mesh $<0.95$. However this value is strongly related to type of physics and the location. FLUENT reports negative cell volumes if volume mesh contains degenerate cells. The develoed model of delfly \& delfly1 shown in fig.4 $\&$ 5.The meshed section of the air craft shown in fig.6. 


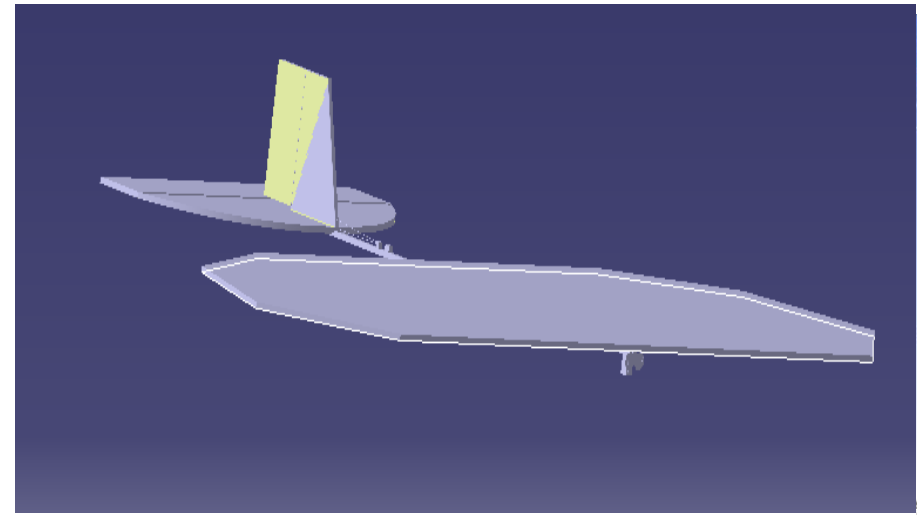

Fig.5 Wing section of delfly 1

\section{Results and Discussion: (Existing Model)}

The analyzed result of deafly 1 in ansys is shown fig.7. From this analyzed results we will be able to understand that where will be the maximum amount of force and parameters are acting. The

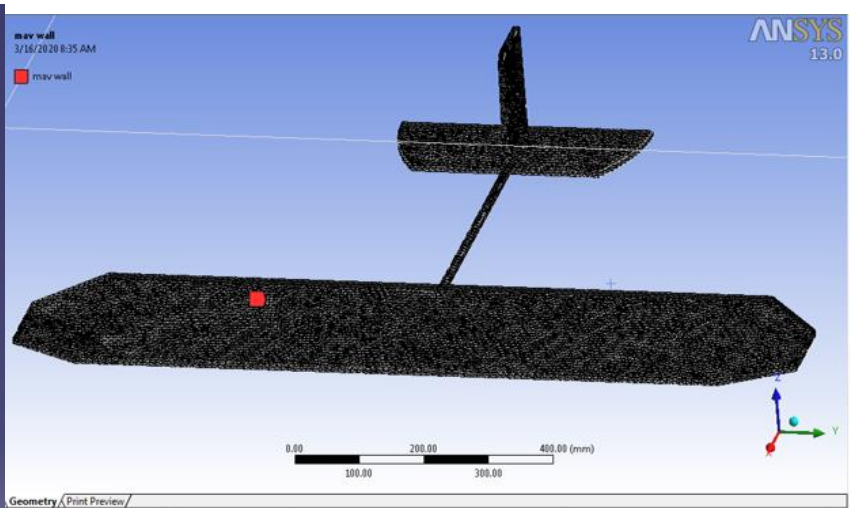

Fig.6.Meshed section of the aircraft fig.7,8 \& 9 shows the velocity countour of the delfly 1.(top view), turbulence kinetic energy (top view) and Velocity contour.

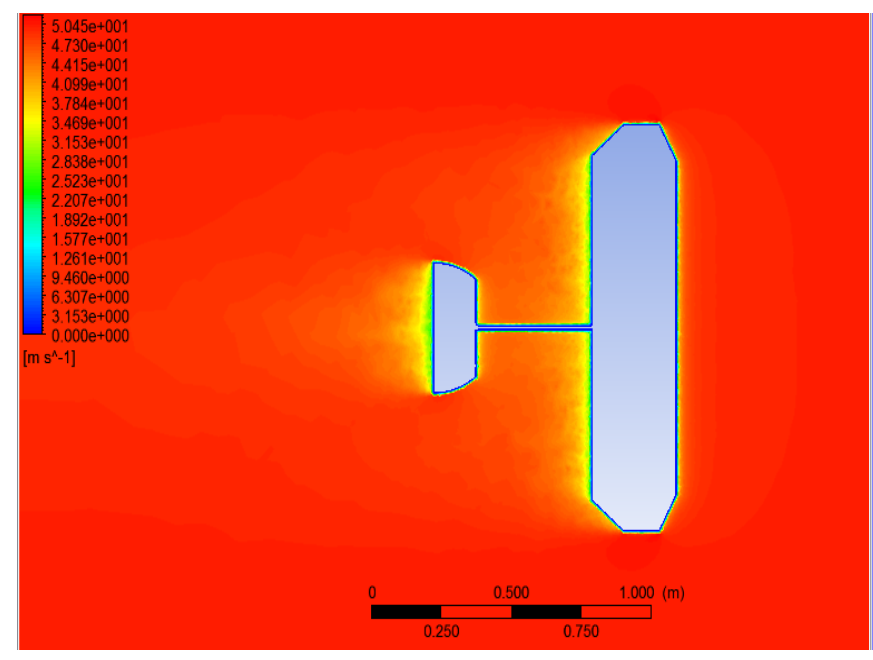

Fig.7.Velocity contours

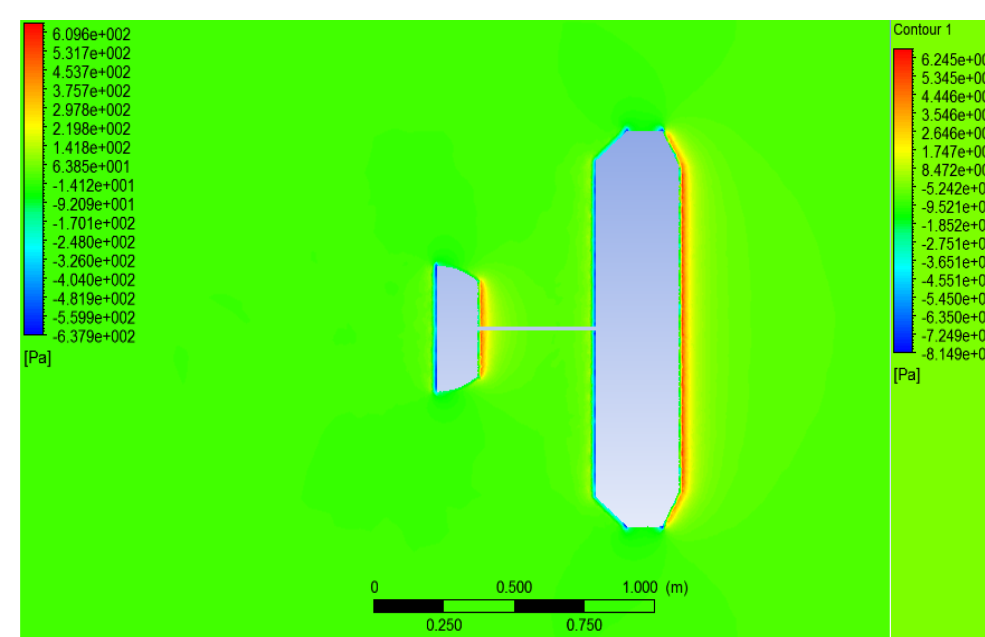

Fig.9.Velocity contour

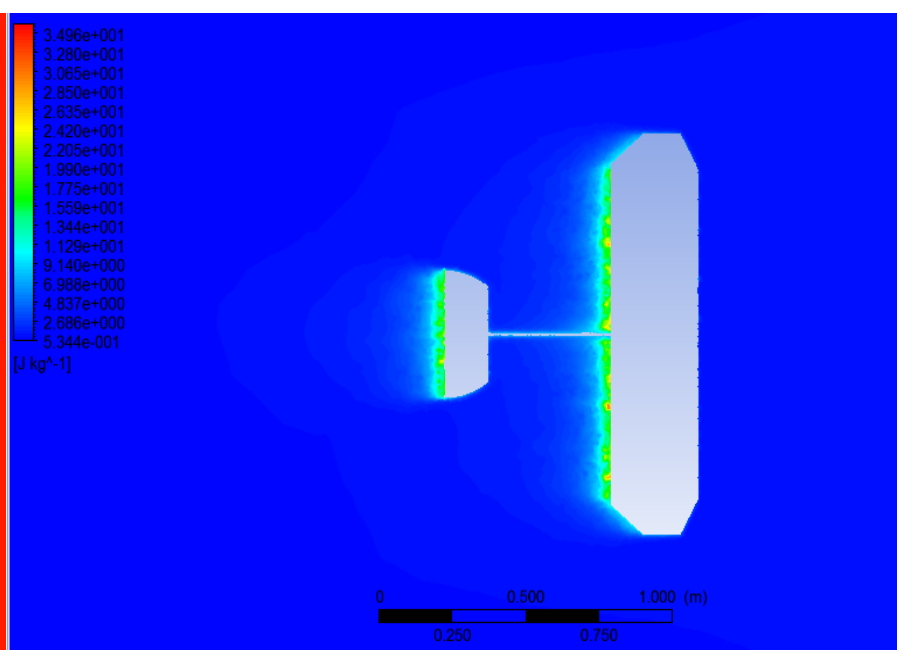

Fig.8. Turbulence contour

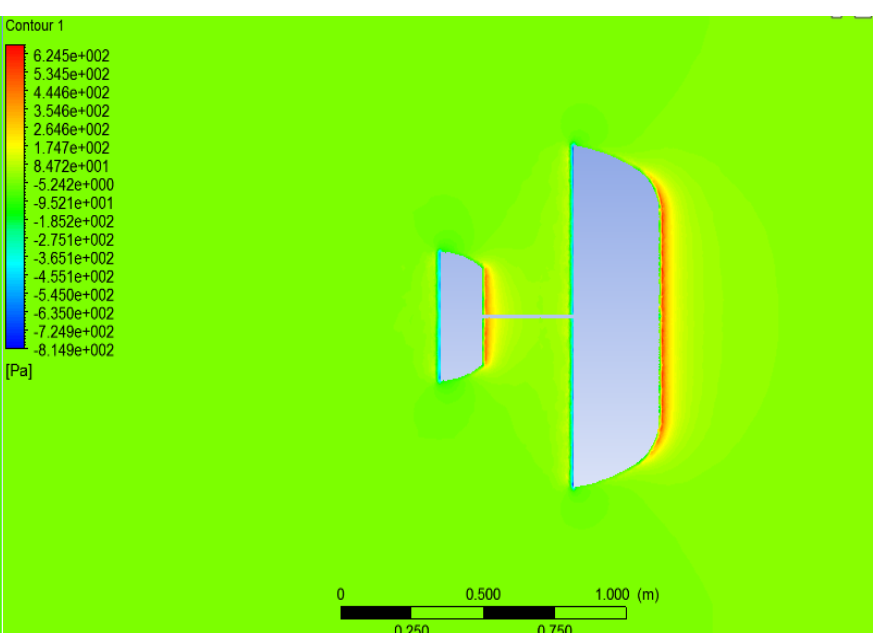

Fig.10. Pressure contour 


\subsection{Results and Discussion (Modified Model)}

By analyzing the delfly 1 model we came to the conclusion that the company is only caring about the lifting capacity of the delfly 1 . so we decided to do some aerodynamic changes in their wing show in Fig.10. The turbulence kinetic enegy (top view) shown in Fig.11. and the velocity contour show in fig. 12 .

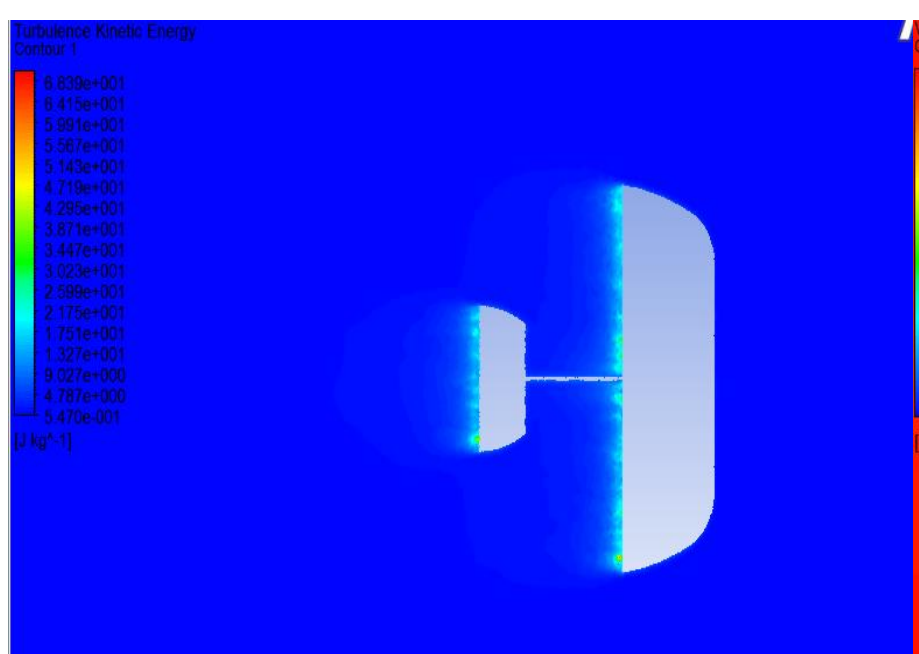

Fig.11.Turbulence contour (modified)

\subsection{Comparative Data of Both Models}

The result we got from the analysis of the modified model and the existing model are plotted below here we made a comparison between the two designs. The plotted diagrams and charts will prove it properly. From the analyzing of the two

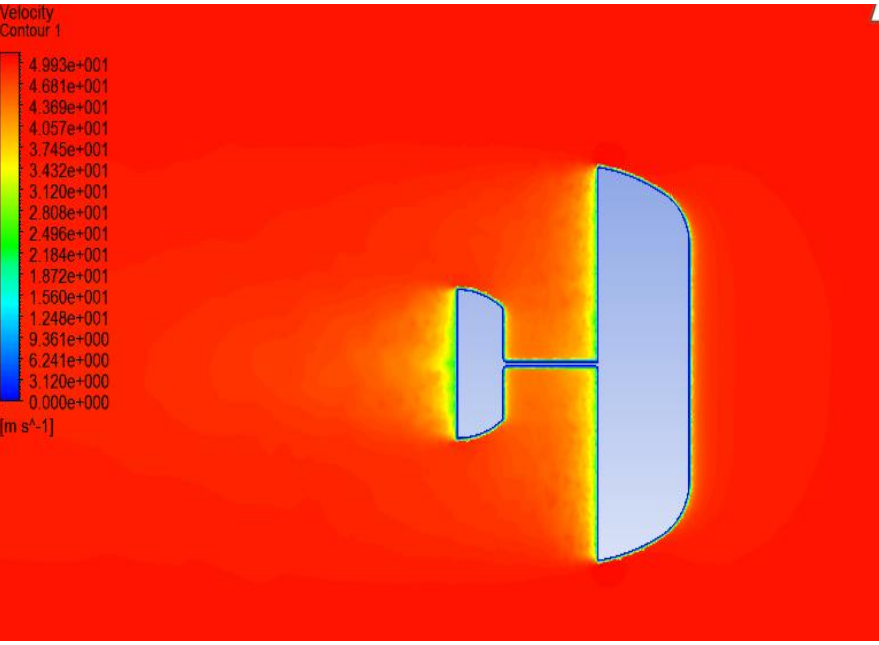

Fig.12. Velocity contour

models we came to know that our model is much more higher performing one when we are comparing to the old delfly model delfly 1 . The Fig.13 shows the comparison between the back pressure \& comparison between the back velocity of the two models.

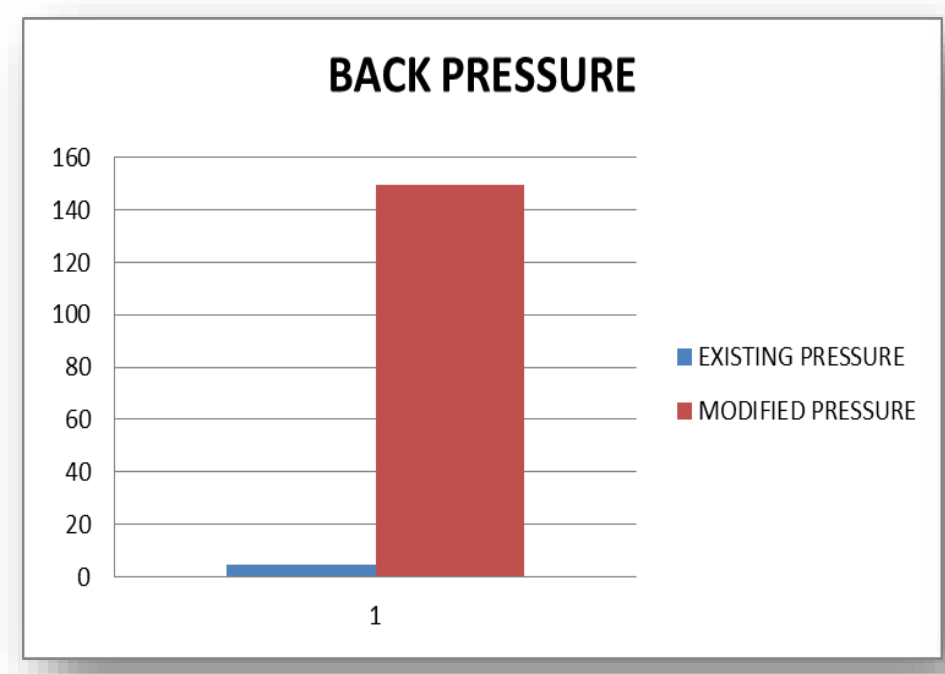

Fig13.Back pressure comparison

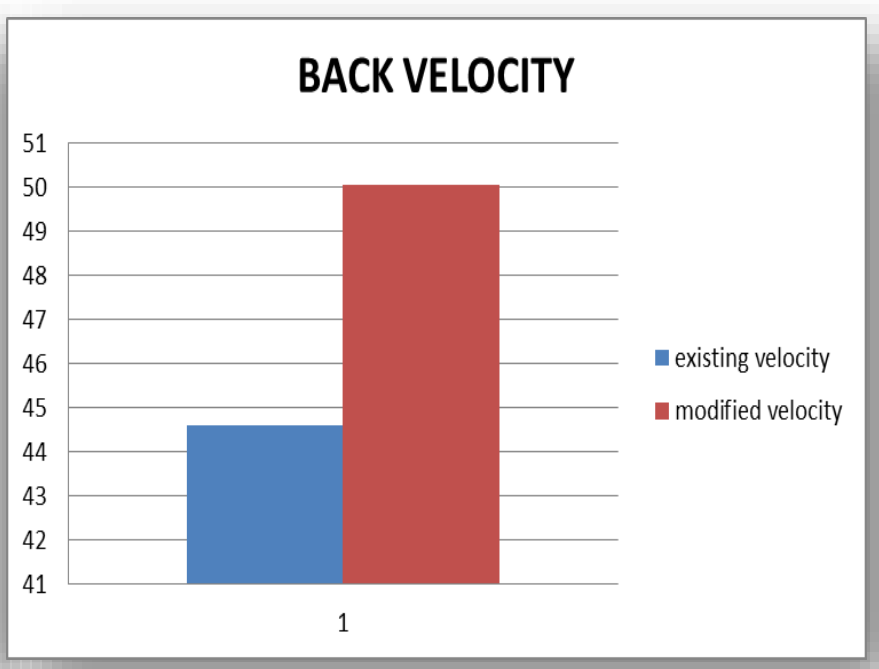

Fig.14 velocity comparison 


\section{Conclusion}

In this project we came across ideas to decrease the surface area and to increase the performance of the mav. Here we took the model of DELFLY-1 and we succeed in it by changing the normal shape wings into an aerodynamic one. As a result of that the drag acting on the MAV become less. Due to this modification the turbulence of the MAV become less, and also the back velocity increased. So in our project we are only aim to improve the speed and to decrease the drag acting on the MAV.

\section{References}

[1] Martin Alexander Jones "CFD Analysis and Design Optimization of Flapping Wing Flows", North Carolina A\&T State University,2013.

[2] W. Shyy, M. Berg, D. Ljungqvist, Flapping and flexible wings for biological and micro air vehicles, Progress in Aerospace Science 35 (1999) 455-505.

[3] M. Sitti, PZT actuated four bar mechanism with two flexible links for micromechanical flying insect thorax, in: IEEE Int. Conf. Robotics and Automation, SouthKorea, 2001, pp. 3893-3900.

[4] B. Tsai, Y. Fu, Design and aerodynamic analysis of a flapping-wing micro aerial vehicle, Aerospace Science and Technology 13 (2009) 383-392.

[5] H. Liu and K. Kawachi. Fixed and Flapping
But in the same delfly there is great chance to improve the lift capacity of the MAV. The lift capacity of the delfly is mainly depend upon its wing. Since it's a flapping type by changing the shape of the wing into something that will produce more drag, we can improve the lifting capacity.If somebody is interested to do more future works in this delfly series we will suggest you to go on with delfly micro, because it is the smallest one in the group.

Wing Aerodynamics for Micro Air Vehicle Applications, volume V-195, chapter "Leading-Edge Vortices of Flapping and Rotary Wings at Low Reynolds Number". AIAA, 2001.

[6] M. A. Mashraf, J. C. S. Lai, and J. Young. Fixed and Flapping Wing Aerodynamics for Micro Air Vehicle Applications, volume V195, chapter "Numerical Analysis of Flapping Wing Aerodynamics". AIAA, 2001.

[7] M. F. Neef and D. Hummel. Fixed and Flapping Wing Aerodynamics for Micro Air Vehicle Applications, volume V-195, chapter "Euler Solutions for a FiniteSpan Flapping Wing". AIAA, 2001.

[8] Jayant Ratti and George Vachtsevanos. Towards energy efficiency in micro hovering air vehicles. In IEEE Aerospace, 2011. 